\title{
Predilection for Junk Food Consumption Among 15-Year-0ld Schoolchildren in North Chennai, India
}

\author{
Shalini Purushothaman ${ }^{1}$, Chandrasekhara Reddy ${ }^{1}$, Preetha E. Chaly ${ }^{1}$, Indra Priyadarshni ${ }^{1}$
}

${ }^{1}$ Department of Public Health Dentistry, Meenakshi Ammal Dental College, Alapakkam main road, Maduravoyal, Chennai - 95, India.

\section{ABSTRACT}

The aim of the present study was to assess the predilection for junk food consumption among 15-year-old schoolchildren in North Chennai.

This was a descriptive cross-sectional study conducted among the schools in North Chennai, India. Cluster sampling methodology was used to recruit the subjects for the study. A questionnaire was used to collect the data. A total of 427, 15-year-old schoolchildren participated in the study. Pearson's chi-square test was performed to determine the significance.

All the participants reported that they liked all types of junk food, and were aware that chemicals were present in the junk food. Nearly $58.3 \%$ of the students knew that junk foods caused diseases. All the participants told that their parents were aware about their junk food consumption habits. Nearly $98.1 \%$ of students reported that the presence of junk food outlets in and around their school influenced them to consume more junk food, which, as reported by all the students, was increasing day by day.

The study concluded that the predilection for junk food is high and the consumption is increasing day- by day among schoolchildren because of various influential factors.

Key Words: Disease, junk food, schoolchildren

\section{INTRODUCTION}

Junk food culture has a vigorously uprising trend among youngsters and it makes people eat without planning. They eat not only during pre-set mealtime, but also in the leisure time $(1,2,3)$. A food that is high in fat, sodium, and/or sugar, provides high calories, but is of no value, is generally known as a "junk food." Junk food seems attractive and is easy to prepare, carry, purchase, and consume. Common junk food includes fast food (burgers, pizzas, patties, nuggets, and so forth), chips, candy, sweet desserts, as well as aerated beverages $(1,4)$. The terms fast food and junk food are often used interchangeably (5). Ingredients of the junk foods give them a good taste, making them addictive, and pose many health hazards.

Multiple factors may influence the consumption of junk food. Studies among adolescents suggest that taste preferences, parental modeling, convenience, marketing strategies, and low prices all may play an influential role $(6,7)$. It is known that food preferences develop in childhood; these dietary behaviors continue into adulthood and increase the risks of chronic diseases later in life $(8,9,10)$. The early stages in the juvenility of teens represent a crucial period of transition in the overall human development; the total nutrient needs are higher during this stage of growth than in any other stage of the life cycle $(11,12)$. It is also the time when a boy or girl increases their social interactions with their peers and develop different eating habits (6). It is a stage when they are least bothered about what they eat (13). Currently, fast food and junk food have expanded their sales in schools (14). For many students, the day is not complete without observing the "daily ritual" of visiting the junk food shop in and around the school premises (5). 
Children are rapidly stepping into the world of junk foods, completely unaware of the havoc they are creating for themselves and the impact on their health (4). However, not much literature is available on perceptions about junk foods among young consumers in Chennai. Keeping in view the predilection for junk food consumption, this study was performed on 15-year-old schoolchildren in North Chennai.

\section{MATERIAL AND METHODS}

Study Design

A descriptive cross-sectional survey was conducted during August to October 2013 among schoolchildren in North Chennai. Ethical approval from the Institutional Review Board and permission from the School Principal were obtained prior to the commencement of the study.

\section{Sample Size}

Following the pilot study, using SPSS Software, and keeping the power of study at $95 \%$ and alpha error at 5\%, the minimum sample size required for the present study was estimated to be 379 subjects.

\section{Sampling Methodology}

Cluster sampling was used to recruit the subjects for the study. The schools in North Chennai formed the clusters. The required sample size was met with three schools.

\section{Study Setting}

The survey was conducted in the classrooms of the randomly selected private schools in Chennai.

\section{Collection of Data}

A pre-tested questionnaire was used to collect the required data. The questionnaire consisted of about 15 closed-ended questions. During the pilot study, the reliability was assessed for randomly selected questions, and on analysis, 99\% agreement was obtained ( $\mathrm{kappa}=0.97$ ). The purpose of the study and the questions in the survey form were clearly explained to the Principal and the class teachers. The teachers then distributed the forms among the students. As the teacher read out each question, the students filled the forms. It took about 5 min to fill the entire form.

\begin{tabular}{llll}
\multicolumn{4}{l}{ TABLE 1: Distribution of study subjects } \\
\hline Schools & No. of subjects & Boys & Girls \\
\hline School 1 & 107 & 68 & 39 \\
School 2 & 173 & 116 & 57 \\
School 3 & 147 & 94 & 53 \\
& 427 & 278 & 149 \\
Total & $(100 \%)$ & $(65.1 \%)$ & $(34.9 \%)$ \\
\hline
\end{tabular}

\section{Statistical Analysis}

The data were entered into MS Excel and processed using Statistical Package for the Social Sciences (IBM SPSS Statistics Version 17). Pearson's chi-square test was used to determine the significance. A P value of $<0.05$ was considered to be statistically significant.

\section{RESULTS}

\section{Distribution of Study Subjects}

The study was conducted in private schools in Chennai. About 427 subjects from three schools participated in the study. The distribution of study subjects is given in Table 1.

\section{Favourite Junk Food}

All the students en masse reported that fast food (burgers, pizzas, nuggets, and so forth), fried snacks (samosa, chips, and so forth), soft drinks (aerated drinks), and confectionaries (candies, pastry, cakes, and ice creams) were their favourite (junk) food items.

\section{Awareness about Junk Food Consumption}

Only 249 students (58.3\%) were aware that junk food consumption caused diseases. About 129 students (30.2\%) reported that they checked the quality of the junk foods prior to consuming them. All the students told that they never checked the nutrient facts label and were aware that chemicals were present in junk foods. Nearly 366 students (85.7\%) reported that they consumed junk food as an alternative to their regular food, and only about 93 students (21.8\%) were ready to substitute their junk food with healthier food items such as fruits and vegetables (Table 2). 


\begin{tabular}{|c|c|c|c|c|}
\hline \multirow[t]{2}{*}{ Question } & $\begin{array}{l}\text { Total } \\
(n=427)\end{array}$ & $\begin{array}{l}\text { Boys } \\
(n=278)\end{array}$ & $\begin{array}{l}\text { Girls } \\
(n=149)\end{array}$ & \multirow[t]{2}{*}{$P$ value } \\
\hline & Yes (\%) & Yes (\%) & Yes (\%) & \\
\hline Junk food causes disease & $\begin{array}{l}249 \\
(58.3)\end{array}$ & $\begin{array}{l}117 \\
(42.1)\end{array}$ & $\begin{array}{l}132 \\
(88.6)\end{array}$ & $<0.001$ \\
\hline Check the quality of food & $\begin{array}{l}129 \\
(30.2)\end{array}$ & $\begin{array}{l}48 \\
(17.2)\end{array}$ & $\begin{array}{l}81 \\
(54.4)\end{array}$ & $<0.001$ \\
\hline Check the nutrient fact label & $0(0)$ & $0(0)$ & $0(0)$ & a \\
\hline Aware that chemicals are present in junk food & $\begin{array}{l}427 \\
(100)\end{array}$ & $\begin{array}{l}278 \\
(100)\end{array}$ & $\begin{array}{l}149 \\
(100)\end{array}$ & a \\
\hline Consume junk food as an alternate to healthy food & $\begin{array}{l}366 \\
(85.7)\end{array}$ & $\begin{array}{l}246 \\
(88.5)\end{array}$ & $\begin{array}{l}120 \\
(80.2)\end{array}$ & $<0.02$ \\
\hline Ready to replace junk food for healthy food & $\begin{array}{l}93 \\
(21.8)\end{array}$ & $\begin{array}{l}0 \\
(100)\end{array}$ & $\begin{array}{l}93 \\
(62.4)\end{array}$ & $<0.001$ \\
\hline Parents are aware of the wards' junk food habits & $\begin{array}{l}427 \\
(100)\end{array}$ & $\begin{array}{l}278 \\
(100)\end{array}$ & $\begin{array}{l}149 \\
(100)\end{array}$ & a \\
\hline
\end{tabular}

\section{Factors Influencing the Choice of Junk Food}

The factors influencing the choice of junk food were time (reported by 212 students [49.6\%]), cost (reported by 377 students [88.3\%]), taste, varieties of junk food, and the influence of advertisements (reported by 427 students [100\%]) (Figure 1).

\section{Influence of Accompanying Person}

About 305 students (71.4\%) reported that they ate junk food items when they were alone and 385 students (90.2\%) ate with their family members. All the students reported that they ate junk food items when they were with their friends (Figure 2). They ate junk food more than three times per day and spent more than hundred rupees per day on these items. Also, their parents were aware about their junk food habits.

The students also stated that junk food outlets were available in and around their school campus, and about 419 students (98.1\%) reported that these junk food outlets influenced them to consume more of these food items.

All the students admitted that their habit of junk food consumption was increasing day by day.

\section{DISCUSSION}

Junk food culture is an emerging trend among the younger generation. Research indicates that junk foods are a potential health threat. The present study has thrown light on the predilection for junk food among the 15-year-old school children in North Chennai.

\section{Awareness about Junk Food Consumption}

In the present study, only 249 students (58.3\%) were aware about the fact that junk food consumption caused diseases. This percentage is almost similar to that reported by Oyedunni et al. (15) (52.1\%) and less compared to the findings of Vinay Gopal et al (1) (85\%) and Aysha (2) (70\%).

Only about 129 students (30.2\%) reported that they would check the quality of the junk food prior to consuming it. This percentage is less compared to a study by Vinay Gopal et al. (1) (57\%).

All the students (100\%) told that they had never checked the nutrient facts label present in the junk food. This percentage is high compared to studies by Vinay Gopal et al. (1) (83\%) and Nurliyana et al. (16) (21.6\%). 


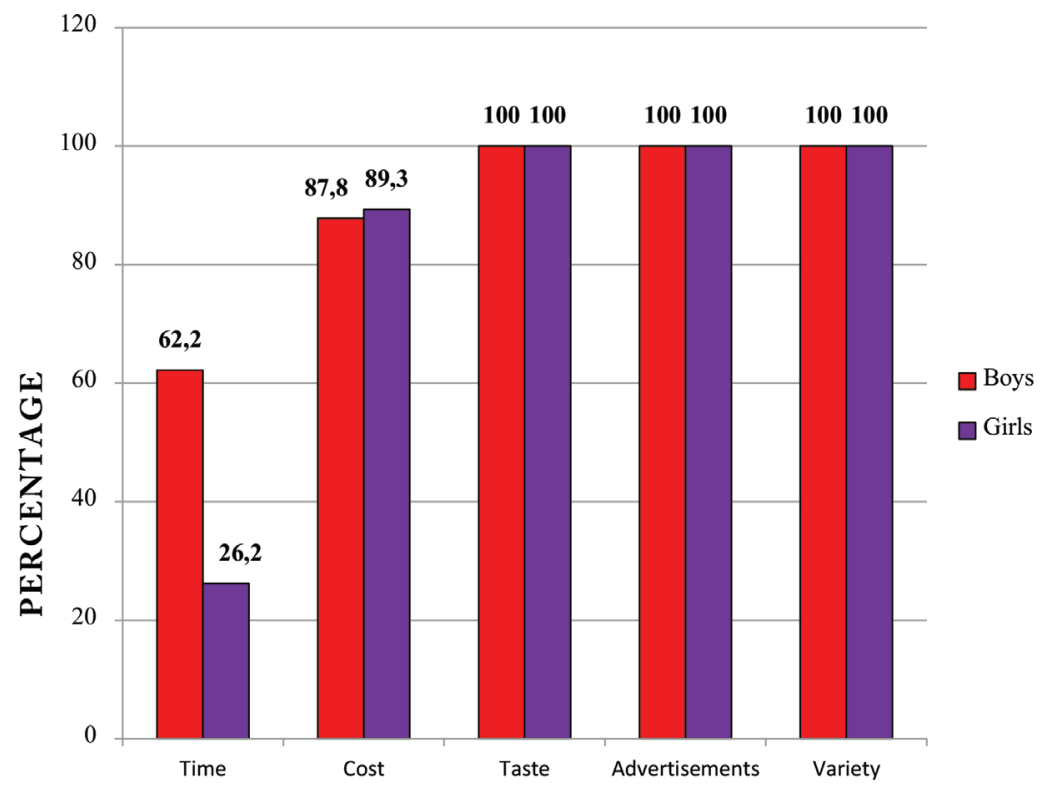

FIGURE 1: Factors influencing the choice of junk food among the subjects.

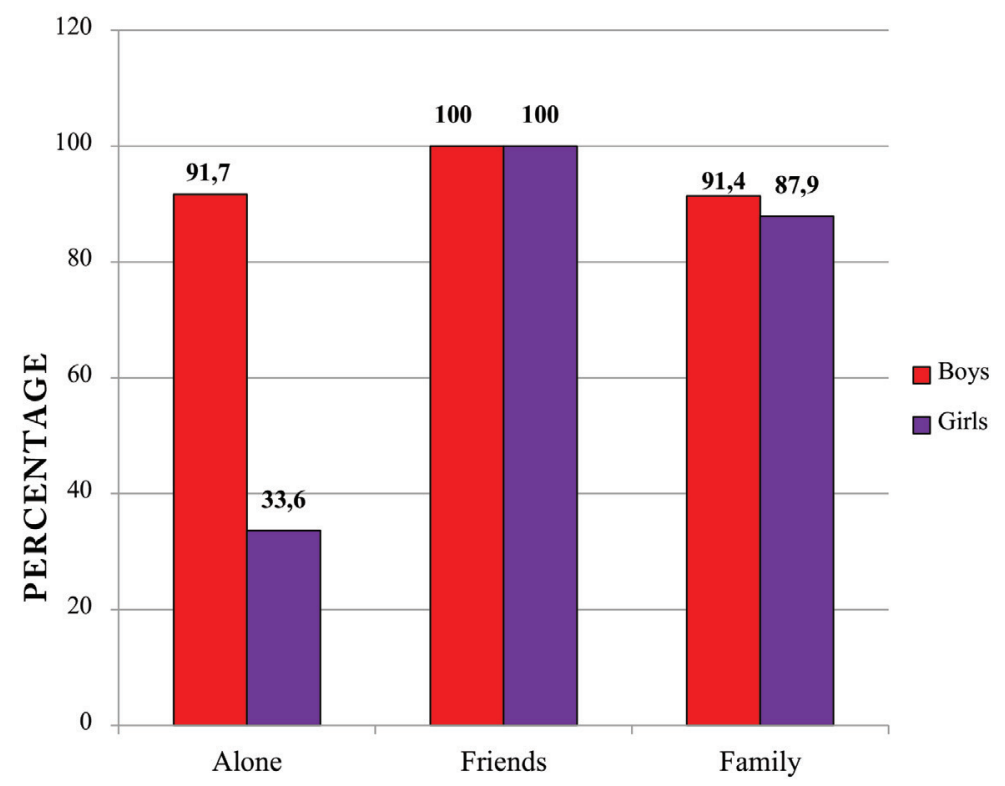

FIGURE 2: Influence of accompanying person in the choice of junk food among the subjects.

All reported that they were aware that chemicals were present in the junk food. This percentage is high compared to a study by Vinay Gopal et al. (1) (33\%).

About 366 students (85.7\%) reported that they consumed junk food as an alternative to their regular food. This percentage is high compared to studies by Vinay Gopal et al. (1) (63\%) and Elizabeth et al. (7) (26.4\%).
Only about 93 students (21.8\%) were ready to substitute their junk food with heal thier food items such as fruits and vegetables. This percentage is less compared to studies by Harsh Kumar et al. (6) (77.1\%) and Oyedunni et al. (15) (93.5\%).

\section{Practices Concerning the Junk Food Consumption}

In the present study, the factors influencing the choice of junk food were time, 212 students (49.6\%); cost, 377 students 
(88.3\%); taste, advertisements, and varieties of junk food, 427 students (100\%). This percentage is varying compared to a study by Naheed Vaida (11) where a majority of the respondents revealed that media was the main attribute for consuming fast foods (80\%), followed by taste (75\%), and variety (33.34\%). Sarah et al. (17) documented that $92.3 \%$ of the respondents preferred junk food because it was quick to cook, $69.2 \%$ preferred it for taste, and $63.6 \%$ because it was inexpensive. Van Zyl et al. (18) in their study, documented that time limitations (58.9\%), convenience (58.1\%), and taste (52.5\%) were the three main reasons for consuming junk food by the subjects.

\section{Influence of Accompanying Person}

In the present study, about 305 students (71.4\%) reported that they ate junk food when they were alone, about 385 students (90.2\%) ate with their family members, and all the students (100\%) reported that they ate junk food items when they were with their friends. This percentage is high compared to a study by Ayse et al. (19) in which 63.5\% of the participants reported that they ate fast food with their friends, $15.3 \%$ with their family, and only about $4.7 \%$ ate alone. Hyun-sun et al. (28) in his study, documented that $61.6 \%$ of the subjects ate fast food with their friends, $18.9 \%$ with their brothers/sisters, $12.7 \%$ with their parents, and only $6.8 \%$ ate fast food when they were alone.

\section{Parents' Awareness about Their Child's Junk Food Habit}

In the present study, all the students (100\%) reported that their parents were aware about their junk food habit. This percentage is high compared to a study by Harsh Kumar et al. (6) (56.9\%).

\section{Junk Food Outlets in and Around School}

In the present study, all the students reported that junk food outlets were present in and around the school campus and about 419 students (98.1\%) told that the presence of junk food outlets in the school influenced them to consume more junk food. Hyun-sun et al. (20) in their study on 354 middle school children in Korea, documented that about 33\% of children consumed junk food only because it was available near the school.

\section{Increase in Junk Food Habit}

All the students (100\%) reported that their habit of junk food consumption was increasing day by day. This percentage is high compared to a study by Vaida (11) (40.96\%).
Goyal and Singh (21) reported that young Indian consumers have more passion for junk food consumption and this predilection is based on three dimensions: service and delivery dimension, product dimension, and quality dimension. Globalization and urbanization have greatly affected an individual's eating habits and, in turn, forced many people to become habituated to the junk food culture. At present, junk food is becoming a proxy to the regular food pattern. Research indicates that unhealthy processed junk foods are a potential health threat, since they contribute to the increased prevalence of obesity. Obesity puts children and adolescents at risk for a range of health problems and can affect cardiovascular health, endocrine system, and mental health.

In spite of these concerns, the present study found a high predilection for junk food consumption among students. This could be because of the time factor, less cost, palatable taste, marketing through a relentless number of advertisements, peer pressure, and easy availability of a wide variety of junk food items. Other factors such as the impact of Western culture leading to a change in the life style, economic status, parental modeling, and convenience of the time-starved families could also play a leading role in increased junk food consumption. The ingredients in the junk foods give them a good taste and make them addictive. The fat and sugar content in these food items is capable of producing dopamine-driven surge of intense pleasure with a propensity for addictive behavior. Free home delivery services, less cost, and easy availability of ready-to-eat packages have a direct proportionality with consumption rates among children, who always want to satisfy their hunger in a fast way. The children are usually curious and experimental, with a wide variety of junk food items readily available to them; hence, junk food consumption is likely to increase. Children are one of the primary targets of food and beverage advertisements; and television continues to be the main form of media for promoting these unhealthy food habits, where celebrities, sports stars, and animated favourite cartoon characters are involved in marketing these products influencing the children in various ways for high consumption of these foods. Findings from several studies indicate that watching television, especially during the high school years, predicts future eating habits. Advertising is a strong and significant predictor of junk food consumption, and because of this, the messages for healthy eating are being undermined. Availability of junk food outlets in the campus and around every 
nook and cranny of schools makes schools a prominent place for junk food consumption. Cafeterias at the schools offer a wide range of junk food to children and this would further increase the junk food consumption. All these factors make children more addicted to these kinds of food items, in turn neglecting healthy food.

\section{CONCLUSION}

The present study is the first to document the predilection for junk food among 15-year-old schoolchildren in North Chennai.

The highlights of the study are as follows:

All the students liked all types of junk food items and their parents were aware of the children's junk food habits. The students were aware that chemicals were present in junk food and its consumption caused diseases. They consumed junk food items as an alternate to regular food, and only a few were ready to replace their junk food with healthy food items.

The time convenience, cost, taste, availability of various types of junk food items, and advertisements were the factors influencing junk food consumption among children.

The presence of junk food outlets in and around the school campus was an influential factor for more consumption, which, as reported by the students, was increasing day by day.

This study contributed to better understanding of the junk food culture among schoolchildren. The study has thrown light on the students' predilection for junk food consumption. It can be concluded from the study that, despite the awareness about the constituents and health threats posed by junk foods, the respondents still engaged in the increasing consumption of these category of foods. The various influential factors included palatable taste, less cost, large variety, easy availability in and around the school premises, and advertisements.

\section{REFERENCES}

1. Gopal V, Sriram, Kannabiran, Seenivasan. Student's perspective on junk foods: Survey. Sudanese Journal of Public Health 2012;7(1):21-25.

2. Baig AK. Review of Trends in Fast Food Consumption. European Journal of Economics, Finance and Administrative Sciences 2012; 48:77-85.

3. Steyn NP, Labadarios $D, N e l ~ J H$. Factors which influence the consumption of street foods and fast foods in South Africa-a national survey.Nutrition Journal 2011;10:104.
4. Ashakiran, Deepthi. Fast Foods and their Impact on Health. JKIMSU 2012; 1(2): 7-15.

5. Kaushik JS, Narang M, Parakh A. Fast Food Consumption in Children.Journal of Indian Pediatrics 2011;48:(2):97-101.

6. Kumar H, Palaha R, Kaur A. Study of consumption, behaviour and awareness of fast food among university hostlers. Asian Journal of Clinical Nutrition 2013;5 (1): 1-7.

7. Denney E, Wilson, Crawford D, Dobbin T, Hardy L, Okely AD. Influences on consumption of soft drinks and fast foods in adolescents. Asia Pac J Clin Nutr 2009;18 (3):447-452.

8. Akman M. Eating patterns of Turkish adolescents: a crosssectional survey. Nutrition Journal 2010; 9:67.

9. Shi Z, Lien N, Kumar BN, Holmboe-Ottesen G. Sociodemographic differences in food habits and preferences of school adolescents in Jiangsu Province, China. European Journal of Clinical Nutrition 2005;59:1439-1448.

10. Salvo D, Frediani JK, Ziegler TR, Cole CR. Food group intake patterns and nutrient intake vary across low-income Hispanic and African American preschool children in Atlanta: a cross sectional study. Nutrition Journal 2012; 11:62.

11. Vaida N. Prevalence of Fast Food Intake among Urban Adolescent Students. The International Journal of Engineering and Science 2013; 2(1): 353-359.

12. Croll JK, Neumark-Sztainer D, Story M. Healthy eating: what does it mean to adolescents. Journal of Nutrition Education 2001; 33(4):193-198.

13. Goyal A, Singh NP. Consumer perception about fast food in India: an exploratory study. British Food Journal 2007; 109(2):182-195.

14. Singla $P$, Sachdeva R, Kochhar A. Effect of nutrition counselling on junk food intake and anthropometric profile among adolescent girls of working mothers. International Journal of Scientific and Research Publications 2012; 2(5):1-7.

15. Oyedunni $\mathrm{S}$ and Modupe $\mathrm{O}$. Fast Food Consumption Pattern among Undergraduates of the University of Ibadan, Nigeria, Implications for Nutrition Education. J Agric Food Tech 2011;1(6): 89-93.

16. Nurliyana G, Norazmir MN, Anuar MK. Knowledge, attitude and practices of university students regarding the use of nutritional information and food labels. Asian Journal of Clinical Nutrition 2011; 3(3):79-91.

17. Rydell SA, Harnack $L$, Oakes M, Story M, Jeffery RW, French SA. Why Eat at Fast-Food Restaurants: Reported reasons among frequent consumers. Journal of the American Dietetic Association 2008; 108(12):2066-2070.

18. Van Zyl MK, Steyn, Marais. Characteristics and factors influencing fast food intake of young adult consumers in Johannesburg, South Africa. S Afr J Clin Nutr 2010;23(3):124-130.

19. Ozcelik AO, Akan LS, Surucuoglu MS. An Evaluation of FastFood Preferences According to Gender. Humanity and Social Sciences Journal 2007;2 (1): 43-50.

20. Seo H, Lee SK, Nam S. Factors influencing fast food consumption behaviors of middle-school students inSeoul: an application of theory of planned behaviours. Nutr Res Pract 2011;5(2):169-178.

21. Goyal A, Singh NP. Consumer perception about fast food in India: an exploratory study. British Food Journal 2007; 109(2): 182-195. 